\title{
Enhanced neophobia induced by incentive contrast
}

\author{
BRUCE R. LOMBARDI \\ Rutgers University, New Brunswick, New Jersey 08903
}

\begin{abstract}
In two experiments, a successive negative contrast effect in licking was produced by shifting rats from $32 \%$ to $4 \%$ sucrose solution. Subsequent to the downshift in reward, the rats were tested for licking either a plain $12 \%$ sucrose solution or $12 \%$ plus a neutral flavor. Licking for the $12 \%$ solution was depressed in downshifted rats when a flavor was present, regardless of whether this flavor was novel or had been present in the shift solution. The results were interpreted in terms of an enhancement of neophobia by reward reduction.
\end{abstract}

Animals that are shifted from a preferred to a less preferred reward generally exhibit depressed performance relative to animals that have had no prior experience with the more preferred reward. This depression of performance, termed a "negative contrast effect," has been found in a wide variety of experiments. For example, negative contrast effects have been found in runway performance with shifts in amount of food (Crespi, 1942; Peters \& McHose, 1974), in errors on complex mazes with shifts to a qualitatively different reward (Elliot, 1928), and in consummatory responding with shifts to a lower concentration of sucrose reward (Lombardi \& Flaherty, 1978; Vogel, Mikulka, \& Spear, 1968). The present experiments investigated the possibility that neophobia and/or conditioned inhibition develop as a consequence of a shift to a less preferred reward.

\section{EXPERIMENT 1}

In Experiment 1, a negative contrast effect was produced in several groups of rats by shifting them from a $32 \%$ to a $4 \%$ sucrose solution. The contrast effect was measured by a depression in licking for $4 \%$ on the part of shifted rats relative to rats that had only received the $4 \%$ solution. For one group of rats in the contrast (downshifted) condition, a neutral flavor was added to the $4 \%$ solution. The flavor present on the contrast day was then added to a $12 \%$ sucrose solution on a subsequent test day. The rationale for this procedure was that if the flavor present in the $4 \%$ solution on the contrast

\footnotetext{
The present report is based on a dissertation submitted in partial fulfillment of the requirements for the PhD degree at Rutgers University. I would like to thank my dissertation advisor, Charles F. Flaherty, for his comments on the present manuscript. I would also like to thank the members of my dissertation committee, Arthur Tomie, Leonard W. Hamilton, George H. Collier, and Carlton T. James. Requests for reprints should be sent to Bruce R. Lombardi, Department of Psychology, University of Maryland Baltimore County, Catonsville, Maryland 21228.
}

day acquired inhibitory properties, licking should be depressed when that flavor was encountered in the sweeter $12 \%$ solution. Another group of rats in the contrast condition received the same sequence of sucrose concentrations (32-4-12), but no flavors were presented. This group was included to control for the possibility that licking a $12 \%$ solution would be depressed as a result of the rats' comparison of the $12 \%$ solution with the sweeter $32 \%$ solution they had received 2 days earlier. A third group of rats was shifted from $32 \%$ to $4 \%$, with a flavor mixed in the $4 \%$ solution, and then received $12 \%$ plus a novel flavor. This control group was included because of recent evidence showing that, in some cases, apparent, associations between poisoning and flavor stimuli are better interpreted as the result of sensitizing the rats' innate avoidance response to novel flavors (Mitchell, 1978; Mitchell, Scott, \& Mitchell, 1977). If poisoning and reward reduction are both aversive events, then reward reduction may also enhance neophobia.

In addition to the three contrast groups outlined above, three groups of rats received only $4 \%$ sucrose solution prior to the $12 \%$ test day. These three groups were given the same flavor treatments as the three contrast groups on the same days, but they experienced no reward reduction. Taken together, these conditions formed a 2 by 3 factorial combination of Preshift Concentration $(32 \%$ vs. $4 \%)$ by Flavor Condition in the test (contrast paired vs. novel vs. plain).

\section{Method \\ Subjects. The subjects were 24 naive male Sprague-Dawley rats purchased from Blue Spruce Farms. All rats were caged individually and maintained on an 8 -h dark/16-h light cycle. The rats were reduced to approximately $80 \%$ of their free-feeding weights and were maintained at that level throughout the experiment by once- a-day feeding and weighing. Daily food rations were provided approximately $1 \mathrm{~h}$ after each day's training session. Water was always available in the home cage.}

Apparatus. The apparatus consisted of six Plexiglas chambers: three measured $23 \times 23 \times 23 \mathrm{~cm}$ and three measured $30 \times 25 \times 25 \mathrm{~cm}$. In one wall of each chamber was a $1.5-\mathrm{cm}$-diam hole $4 \mathrm{~cm}$ above 
the floor. Outside the chamber, a graduated cylinder was mounted so that the orifice of a drinking spout was centered in the hole, flush with the outside wall of the chamber. A wire was immersed in the solution contained in the cylinder and was connected to a drinkometer circuit that was completed through the grid floor of the apparatus each time the animal made contact with the solution. Licks were recorded by a Data General Nova 2 computer that recorded licks in 1-min bins for 5 min starting with an animal's first lick. Each apparatus was enclosed in a sound-attenuating structure.

Procedure. The rats were assigned randomly to one of six groups $(n=4)$. Throughout the experiment, the rats were allowed $5 \mathrm{~min}$ access per day (timed from the first lick) to the appropriate sucrose solution in the test apparatus. In the preshift phase, which lasted 11 days, three of the six groups received $4 \%$ sucrose solution, while the remaining groups received $32 \%$. On Day 12 , the contrast day, all groups received $4 \%$, with a flavor added if specified by their condition. On Day 13 , the test day, all groups were tested with $12 \%$ plus the appropriate flavor. The individual groups were coded as follows: $32-4 f-12 f ; 32-4 f-12 n f ; 32-4-12$; $4-4 f-12 f ; 4-4 f-12 n f ; 4-4-12$. The first number refers to the sucrose concentration received during preshift training, the second number refers to the sucrose concentration received on the contrast day, and the third number refers to the concentration received on the test day. The letters $f$ and $n f$ stand for flavor and novel flavor, respectively. For example, Group $32-4 \mathrm{f}-12 \mathrm{nf}$ received $32 \%$ during preshift, followed by $4 \%$ plus a flavor on the contrast day, followed by $12 \%$ plus a novel flavor on the test day.

The sucrose solutions were made from commercial grade sugar and tap water with the percentage calculated by weight (e.g., $32 \%_{0}=32 \mathrm{~g}$ sugar $+68 \mathrm{~g}$ water). McCormick's orange and vanilla exiracts were used as flavors and were counterbalanced as either contrast paired or novel. The flavors were added to the solutions by substituting $2.5 \mathrm{ml}$ of the appropriate flavor for $2.5 \mathrm{ml}$ of water, thereby maintaining the same sugar concentration as in the plain sucrose solutions. All solutions were made fresh each day, $24 \mathrm{~h}$ prior to their use in testing.

\section{Results}

An analysis of variance conducted on the preshift data, averaged over the last 3 preshift days, revealed that all groups receiving $32 \%$ licked at a higher rate than those receiving $4 \%[F(1,18)=11.25, \mathrm{p}<.01]$. No significant differences among the groups receiving $32 \%$ or among the groups receiving $4 \%$ were present (Flavor Condition and Flavor Condition by Preshift Concentration, Fs $<1$ ).

On the contrast day, all groups that were shifted from $32 \%$ to $4 \%$ licked less than the unshifted groups $[F(1,18)=15.92, p<.001]$ and therefore exhibited a negative contrast effect. Neither the negative contrast effect nor the licking of the noncontrast groups were affected by the introduction of a flavor (Flavor Condition and Flavor Condition by Preshift Concentration, Fs $<1$ ).

The data from the $12 \%$ test day are presented in Figure 1. The left panel shows the mean number of licks as a function of minute for the contrast groups, and the right panel shows the same data for the noncontrast groups. The figure shows that the contrast groups that received either a contrast-paired or novel flavor in the test licked at a similar rate that was less than that of the contrast group receiving plain $12 \%$. The depression in these groups is most apparent in the first few minutes of testing. These groups were also
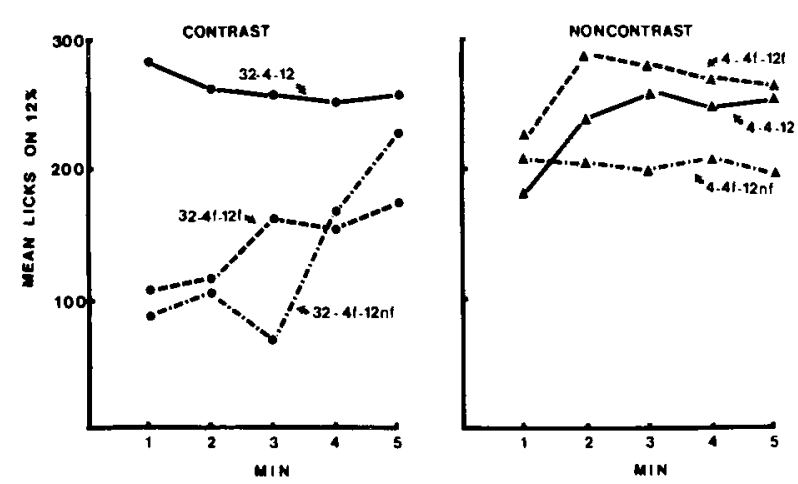

Figure 1. Mean licks for each condition as a function of minute on the test day of Experiment 1.

licking at a lower rate than the noncontrast groups. The contrast group that received plain $12 \%$, however, licked at a rate equivalent to that of the noncontrast groups.

The patterns apparent in the figure were confirmed statistically by an analysis of variance, which revealed a reliable Preshift Concentration by Flavor Condition by Minute interaction $[F(8,72)=2.27, p<.05]$. Subsequent analysis of this interaction with Fisher's LSD procedure revealed that in minutes 1 and 2 of testing, both the contrast group receiving the novel flavor and the contrast group receiving the contrast-paired flavor licked at a similar rate that was reliably less than all other groups. There were no reliable differences among any other groups.

\section{Discussion}

Rats that experienced a shift to a less preferred reward licked less for flavored $12 \%$ sucrose solution than either rats that did not experience reward reduction or rats that experienced the reward reduction but received plain $12 \%$ in the test. Since the same-flavor and novel-flavor contrast groups showed equal reduction, it cannot be concluded that the reduction was due to the association of the flavor with the shift (cf. Hearst, 1972). These results suggest that the effect of the reduction in reward was to sensitize the rats' innate avoidance response to novel flavors, a response that has been termed "neophobia" (Barnett, 1963; Mitchell, 1976). The sensitization or enhancement of neophobia in rats has been reported following poisoning (Carroll, Dinc, Levy, \& Smith, 1975; Rzoska, 1953) and appears to have occurred following negative contrast in the present experiment.

\section{EXPERIMENT 2}

An important observation in Experiment 1 was that rats receiving a novel flavor in the test following negative contrast licked at a reduced rate. This observation was taken as evidence that the contrast effect produced enhanced neophobia. However, an interpretation based on generalization of inhibition 
would also seem plausible, since the contrast rats that received a novel flavor on the test day had received a different flavor on the contrast day. That is, if the flavor present on the contrast day had acquired inhibitory properties, and there was some similarity between that flavor and the novel flavor, the reduced licking in the test may have been due to generalization of the acquired inhibitory properties to the novel flavor. As a means of deciding between these two explanations, a second experiment was conducted that included a group shifted from $32 \%$ to plain $4 \%$ prior to being tested with a novel flavor. Since this group did not receive a flavor on the contrast day, there should be no opportunity for generalization of inhibition. If reduced licking were observed in this group, the neophobia explanation would be favored; an absence of reduced licking would favor a generalization of inhibition interpretation.

Experiment 2 also provided a partial replication of Experiment 1 (partial because the only noncontrast group was one that received a novel flavor in the test) and also included a group that was shifted from $32 \%$ to flavored $4 \%$ and was then tested with plain $12 \%$.

\section{Method}

Subjects. The subjects were 60 naive male Sprague-Dawley rats purchased from Blue Spruce Farms. The rats were reduced to approximately $80 \%$ of their free-feeding body weights and were housed and maintained as in Experiment 1. The apparatus was also the same as that used in Experiment 1.

Procedure. The rats were assigned randomly to one of six groups $(n=10)$. The procedure was similar to that of Experiment 1 except for the following modifications: During the preshift period (12 days), only one of the six groups received $4 \%$ and the remaining groups received $32 \%$. On the contrast day, all subjects received $4 \%$ plus a flavor if applicable, and then, on the test day, all subjects received $12 \%$ plus the appropriate flavor. The groups and flavor conditions, designated as in Experiment 1, were as follows: 32-4-12, 32-4f-12f, 32-4f-12nf, 32-4f-12, 32-4-12nf, and 4-32-12nf. The two flavors were prepared and counterbalanced as in Experiiment I, but anise (McCormick's anise extract) was used instead of vanilla.

\section{Results}

No reliable differences in licking existed among the groups during the preshift period $(\mathrm{F}<1)$. An analysis of variance conducted on the data from the contrast day showed a significant Group effect $[F(5,54)=23.33$, $\mathrm{p}<.001$ ], and subsequent analysis with Fisher's LSD revealed that all shifted groups licked less than the unshifted group (a negative contrast effect). The lick rates of the downshifted groups did not differ reliably from one another.

The results of the $12 \%$ test day are presented in Figure 2. The figure shows mean licks on $12 \%$ as a function of minute, with the data for each group in a separate panel. The data for the three contrast groups that received flavors in the test are presented in the left panels. The lick rates of these groups appear similar across minutes and lower than those of
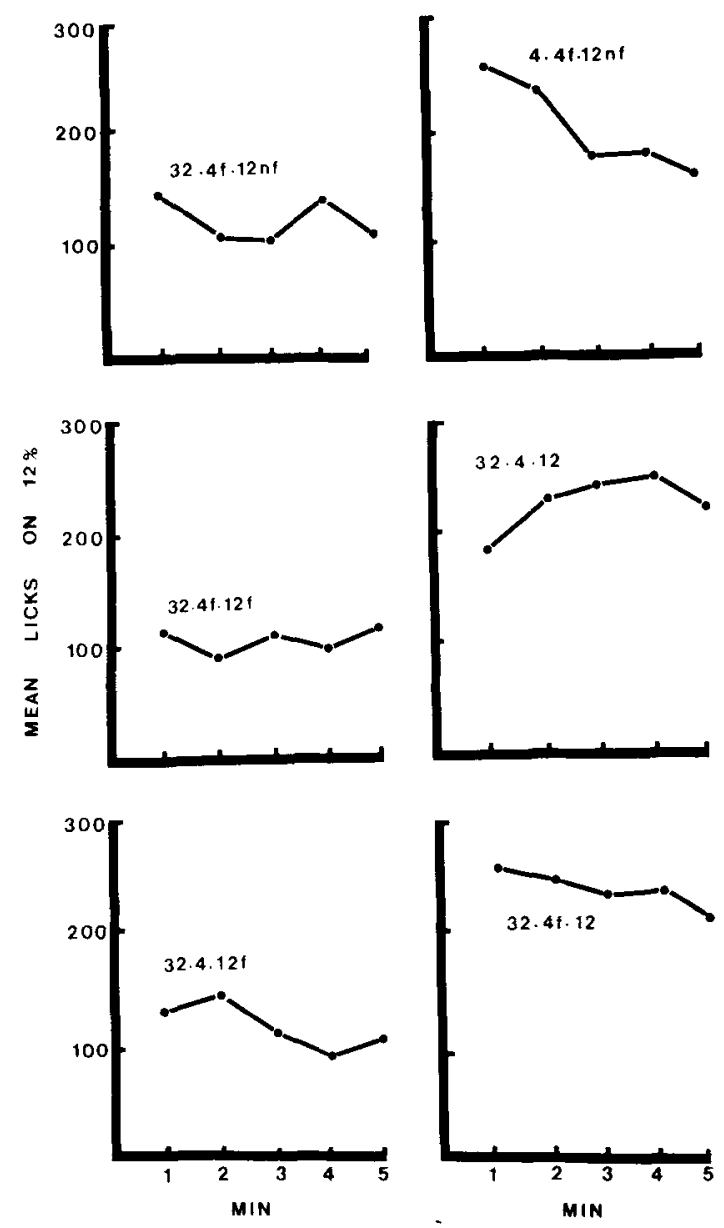

Figure 2. Mean licks for each condition as a function of minute on the test day of Experiment 2.

the noncontrast group (upper right panel). The lick rates of these groups also appear lower than those of the contrast groups receiving plain $12 \%$ on the test day (two lower right panels).

An analysis of variance of these data revealed a reliable Group effect $[\mathrm{F}(5,54)=9.86, \mathrm{p}<.001]$, and subsequent analysis of this effect with Fisher's LSD showed that the patterns apparent in the figure were statistically reliable. The analysis of variance also revealed a reliable Group by Minute interaction $[\mathrm{F}(20,216)=2.38, \mathrm{p}<.01]$. This effect appears to be due mainly to the decline in licking exhibited by the noncontrast group (4-4f-12nf).

\section{Discussion}

The results of Experiment 2 generally replicated those obtained in Experiment 1. Rats that experienced negative contrast exhibited a depression in licking when either a novel or contrast-paired flavor was encountered in the test solution. However, the downshift in concentration per se did not result in reduced licking on plain 12\%, since neither Group 32-4-12 nor Group 32-4f-12 licked less than the noncontrast group. These results preclude the explanation that 
the depressed licking on the part of contrast rats receiving flavors in the test was due simply to their comparing the $12 \%$ solution with the sweeter solution they received 2 days earlier.

An important finding of this experiment was that contrast rats receiving a flavor for the first time on the test day (Group 32-4-12f) showed a depression in licking equivalent to that of the group that received one flavor on the contrast day and a different flavor on the test day $(32-4 f-12 n f)$. This result provides evidence against the possibility that the reduced licking in the presence of a novel flavor was due to generalization of inhibition. The simplest explanation for the equivalence of these two groups seems to be that the contrast effect sensitized the rats' neophobic response to novel flavors.

If flavor novelty is important in determining the depressed licking, then the depression shown by Group $32-4 \mathrm{f}-12 \mathrm{f}$ presents a problem; that is, the test-day flavor was not new. However, it is reasonable to assume that the flavor was still relatively novel on the test day, since it was only the rats' second experience with it.

Taken together, the results of both Experiments 1 and 2 support the view that a shift to less favorable reward conditions produces enhanced neophobia. This finding suggests that a downshift in reward and manipulations such as poisoning, which also produce enhanced neophobia, have something in common. The common basis may be physiological, since both reward reduction and the poisons that produce neophobia and conditioned aversions increase the plasma levels of the stress-related hormone, corticosterone (see Goldman, Coover, \& Levine, 1973, for the data on reward reduction, and Braveman, 1977, for a discusssion of the data on poisons).

\section{REFERENCES}

Bannett, S. A. The rat: A study in behavior. Chicago: Aldine, 1963.
Braveman, N. S. What studies on pre-exposure to pharmacological agents tell us about the nature of the aversion inducing treatment. In L. M. Barker, M. R. Best, \& M. R. Domjan (Eds.), Learning mechanisms in food selection. Waco, Tex: Baylor University Press, 1977.

Carroll, M. E., Dinc, H. I., Levy, C. J., \& Smith, J. C. Demonstrations of neophobia and enhanced neophobia in the albino rat. Journal of Comparative and Physiological Psychology, 1975, 80, 457-467.

Crespl, L. P. Quantitative variations of incentive and performance in the white rat. American Journal of Psychology, 1942, 55, 467-517.

Elliot, M. H. The effect of change of reward on the maze performance of rats. University of California Publications in Psychology, 1928, 4, 19-30.

Goldman, L., Coover, G. D., \& Levine, S. Bidirectional effects of reinforcement shifts on pituitary adrenal activity. Physiology \& Behavior, 1973, 10, 209-214.

HEARST, E. Some persistent problems in the analysis of conditioned inhibition. In R. A. Boakes \& M. S. Halliday (Eds.), Inhibition and learning. New York: Academic Press, 1972.

Lombardi, B. R., \& Flaherty, C. F. Apparent disinhibition of successive but not of simultaneous negative contrast. Animal Learning \& Behavior, 1978, 6, 30-42.

Mitchell, D. Experiments on neophobia in wild and laboratory rats: A reevaluation. Journal of Comparative and Physiological Psychology, 1976, 90, 190-197.

Mitchels, D. The psychological vs. the ethological rat: Two views of the poison avoidance behavior of the rat compared. Animal Learning \& Behavior, 1978, 6, 121-124.

Mitchell, D., Scotт, D. W., \& Mitchell, L. K. Attenuated and enhanced neophobia in the taste-aversion "delay of reinforcement" effect. Animal Learning \& Behavior, 1977, 5, 99-102.

Peters, D. P., \& McHose, J. H. Effects of varied preshift reward magnitude on successive negative contrast effects in rats. Journal of Comparative and Physiological Psychology, 1974, 86, 85-95.

Rzoska, J. Bait shyness, a study in rat behavior. British Journal of Animal Behaviour, 1953, 1, 128-135.

Vogel, J. R., Mikulka, P. J., \& Spear, N. E. Effects of shifts in sucrose and saccharine concentrations on licking behavior in the rat. Journal of Comparative and Physiological Psychology, $1968,66,661-666$.

(Received for publication July 12, 1979; revision accepted June 5,1980 .) 